\title{
Unconstrained nonlinear MPC: Performance Estimates for Sampled-data Systems with zero order hold
}

\author{
Karl Worthmann ${ }^{1}$, Marcus Reble ${ }^{2}$, Lars Grüne ${ }^{3}$, and Frank Allgöwer ${ }^{2}$
}

\begin{abstract}
In this paper, model predictive control (MPC) schemes without stabilizing terminal constraints and/or costs are considered for continuous time systems governed by ordinary differential equations. Satisfactory estimates of the required prediction horizon length such that the MPC closed loop is asymptotically stable were recently proposed. However, their applicability is, in general, limited by the fact that the respective proofs require possible discontinuities of the input functions at arbitrary (and a priori unknown) switching times. We present a technique which allows to determine a suitable discretization accuracy such that the obtained performance bound is arbitrarily well recovered for sampled-data systems with zero order hold.
\end{abstract}

\section{INTRODUCTION}

Model predictive control (MPC) is nowadays a well established technique in order to stabilize a system at a desired set point — both in theory and practice, see [12] and [10], [15], [17]. In MPC, the control problem formulated on an infinite time horizon is approximated by a sequence of finite horizon optimal control problems (OCPs), which are iteratively solved. However, the stability analysis is far from being trivial, see, e.g., [11]. One option is to incorporate additional (artificial) terminal constraints and/or costs in the OCPs to be solved in each MPC step, see, e.g., [2], [12]. Alternatively, under a controllability condition the prediction (optimization) horizon of these OCPs can be chosen sufficiently large, cf. [6]. Recently, techniques to determine the length of the prediction horizon such that stability is ensured were proposed, see [8] for linear and [16], [4] for nonlinear systems. In addition to stability guarantees, these methodologies yield performance estimates of the MPC closed loop compared to an infinite horizon optimal trajectory.

In this paper, the focus is put on continuous time systems governed by ordinary differential equations. In order to apply the existing results, two ways are possible. On the one hand, the system dynamics can be represented as a sampled-data system in order to treat them within the framework proposed in [16], [4]. On the other hand, the genuine continuous time approach as carried out in [13] yields significantly

\footnotetext{
${ }^{1}$ Karl Worthmann is with the Institute for Mathematics, Technische Universität Ilmenau, 98693 Ilmenau, Germany, karl.worthmannetu-ilmenau. de

${ }^{2}$ Frank Allgöwer and Marcus Reble are with the Institute for Systems Theory and Automatic Control, University of Stuttgart, 70550 Stuttgart, Germany, [frank.allgoewer, marcus.reble] aist.uni-stuttgart.de

${ }^{3}$ Lars Grüne is with the Mathematical Institute, University of Bayreuth, 95440 Bayreuth, Germany, lars . gruene@uni-bayreuth. de

This paper is a substantially revised version of [19] whose submission to the CDC was encouraged by SIAM.
}

better (performance) estimates at the expense of using $\mathcal{L}_{\mathrm{loc}^{-}}^{1}$ functions in the MPC algorithm and, thus, not being valid for inputs which are piecewise constant on equidistant intervals, as generated by zero order hold sampling. This even holds if the invoked controllability condition is established for control functions which are piecewise constant on equidistant intervals. We propose a mechanism such that the superior performance bounds resulting from [13] are maintained for sampled-data systems with zero order hold. To this end, decoupling the sampling rate (discretization) and the control horizon in MPC is essential. Moreover, the concept of multistep feedback laws plays a vital role in the approach.

The outline of the present paper is as follows. First, in Section II the problem formulation and performance bounds for MPC based on a controllability condition are revisited. Then, in Section III important tools such as multistep feedback laws and discretizations are introduced. The main results are presented in Section IV and conclusions are drawn in Section V. During all of these sections, the results are illustrated with a nonholonomic integrator example.

Notation: The natural and real numbers are denoted by $\mathbb{N}$ and $\mathbb{R}$, respectively. For $r \in \mathbb{R},\lfloor r\rfloor$ stands for the largest integer $\leq r$. The class $\mathcal{K}_{\infty}$ contains all continuous functions $\eta: \mathbb{R}_{\geq 0} \rightarrow \mathbb{R}_{\geq 0}$, which are zero at zero, strictly monotonically increasing, and unbounded. Let $I \subset \mathbb{R}$ be an interval. A function $u: I \rightarrow \mathbb{R}^{m}$ is said to be piecewise continuous - denoted by $\mathcal{P C}\left(I, \mathbb{R}^{m}\right)$ - if, for every $a, b \in I$ with $a<b$, the interval $[a, b]$ admits a finite partition $a=$ $t_{1}<t_{2}<\cdots<t_{n}=b, n \in \mathbb{N}$, such that $u$ is continuous on every subinterval $\left(t_{i}, t_{i+1}\right), i \in\{1, \ldots, n-1\}$, has a right $\operatorname{limit}_{\lim _{t} t_{1}} u(t)$ at $t_{1}$, a left $\operatorname{limit}_{\lim _{t} \nearrow_{n}} u(t)$ at $t_{n}$, and both left and right limit at every $t_{i}, i \in\{2,3, \ldots, n-1\}$, see [7, p.17]. Moreover, a function $u: I \rightarrow \mathbb{R}^{m}$ belongs to $\mathcal{L}_{\text {loc }}^{1}\left(I, \mathbb{R}^{m}\right)$ if its restriction to every compact interval $K \subset I$ is measurable and satisfies $\int_{K}\left|u_{i}(t)\right| d t<\infty$ for all $i \in$ $\{1,2, \ldots, m\}$, see, e.g. [14, p.471].

\section{MPC FOR CONTINuOUS TIME Systems}

We consider a continuous time system governed by the ordinary differential equation

$$
\dot{x}(t)=f(x(t), u(t))
$$

with continuous vector field $f: \mathbb{R}^{n} \times \mathbb{R}^{m} \rightarrow \mathbb{R}^{n}$, which is locally Lipschitz continuous with respect to its first argument. Here, $x(t) \in \mathbb{R}^{n}$ and $u(t) \in \mathbb{R}^{m}$ represent the state and the control input at time $t \in \mathbb{R}_{\geq 0}$, respectively. For given state $x^{0} \in \mathbb{R}^{n}$ and control function $u \in \mathcal{L}_{\text {loc }}^{1}\left(\mathbb{R}_{\geq 0}, \mathbb{R}^{m}\right)$ the solution is denoted by $x\left(\cdot ; x^{0}, u(\cdot)\right)$ on its maximal interval 
of existence. The control values are confined to a closed set $\mathbb{U} \subseteq \mathbb{R}^{m}$ with nonempty interior int $\mathbb{U}$. Furthermore, let $\left(x^{\star}, u^{\star}\right) \in \mathbb{R}^{n} \times \operatorname{int} \mathbb{U}$ be such that $f\left(x^{\star}, u^{\star}\right)=0$ holds. Then, $x^{\star} \in \mathbb{R}^{n}$ is called a controlled equilibrium, i.e., $x\left(t ; x^{\star}, u(\cdot)\right)=x^{\star}$ for all $t \geq 0$ with $u(\cdot) \equiv u^{\star}$.

\section{A. Model Predictive Control}

Our goal is to stabilize system (1) at the (controlled) equilibrium $x^{\star}$. Since we want to fulfill this control task in an optimal fashion, stage costs $\ell: \mathbb{R}^{n} \times \mathbb{R}^{m} \rightarrow \mathbb{R}_{\geq 0}$ satisfying the conditions

$$
\ell\left(x^{\star}, u^{\star}\right)=0 \quad \text { and } \quad \ell(x, u) \geq \underline{\eta}\left(\left\|x-x^{\star}\right\|\right)
$$

with a $\mathcal{K}_{\infty}$-function $\eta$ are employed as a performance criterion. Then, the following MPC scheme with prediction horizon $T \in \mathbb{R}_{>0}$ and control horizon $\delta \in(0, T)$ is used.

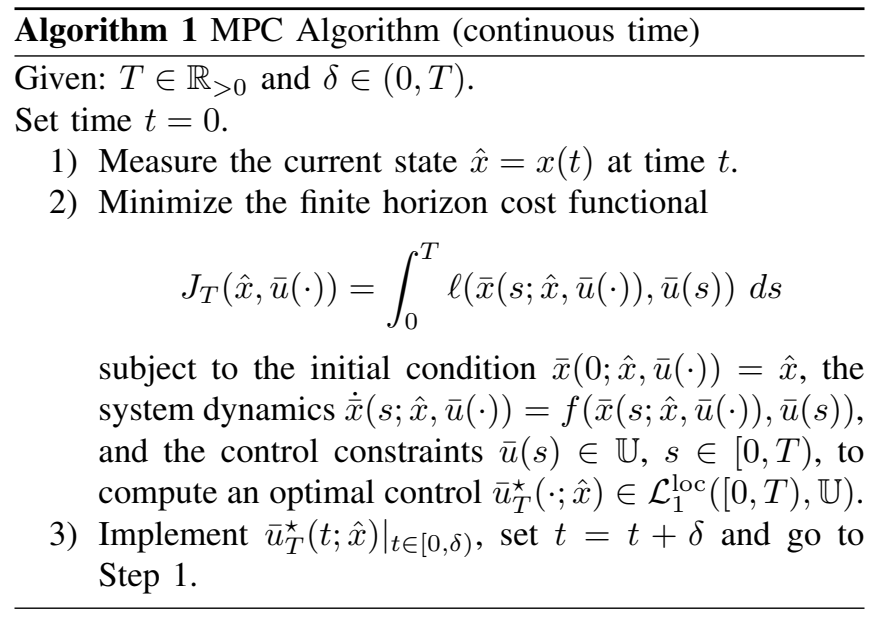

Remark 1 (Existence of a minimizer) In Step 2 of Algorithm 1 it is tacitly assumed that the infimum

$$
V_{T}(\hat{x}):=\inf _{\bar{u}(\cdot)} J_{T}(\hat{x}, \bar{u}(\cdot)),
$$

is attained, i.e. $V_{T}(\hat{x})=J_{T}\left(\hat{z}, \bar{u}_{T}^{\star}(\cdot ; \hat{x})\right)$ holds, without specifying any regularity properties of $\bar{u}:[0, T) \rightarrow \mathbb{R}^{m}$. A likely choice would be the space of piecewise continuous (constant) functions. Then, the infimum may be an element of $\mathcal{L}_{1}^{\text {loc }}\left([0, T), \mathbb{R}^{m}\right)$ since $\mathcal{P C}\left([0, T), \mathbb{R}^{m}\right)$ is not closed.

The procedure described in Algorithm 1 allows to define a state feedback law $\mu_{T, \delta}:[0, \delta) \times \mathbb{R}^{n} \rightarrow \mathbb{U}$ as $\mu_{T, \delta}(s, \hat{x}):=\bar{u}_{T}^{\star}(s ; \hat{x})$, i.e. the control loop is closed every $\delta$ time units. The resulting (nominal) MPC closed loop trajectory $x_{\mu_{T, \delta}}^{M P C}\left(\cdot ; x_{0}\right)$ is generated iteratively by

$\dot{x}_{\mu_{T, \delta}}^{M P C}(t)=f\left(x_{\mu_{T, \delta}}^{M P C}(t), \mu_{T, \delta}\left(t-\lfloor t / \delta\rfloor \delta, x_{\mu_{T, \delta}}^{M P C}(\lfloor t / \delta\rfloor \delta)\right)\right)$ with $x_{\mu_{T, \delta}}^{M P C}(0)=x^{0}$. The control function consisting of the concatenated input signals is denoted by $u_{\mu_{T, \delta}}^{M P C}\left(\cdot ; x^{0}\right)$. For further details and explanations on nonlinear MPC we refer to [3] and the references therein.

Remark 2 (Extensions) Algorithm 1 and also the upcoming results can be generalized to infinite dimensional systems including state constraints, see [19] for details.

\section{B. Performance Estimates \& Asymptotic Stability}

Asymptotic stability of this MPC scheme can be ensured by the following theorem [13]. The idea of using a relaxed Lyapunov inequality as a sufficient condition in order to guarantee asymptotic stability of a receding horizon control scheme goes back to [5].

Theorem 1 Assume existence of a suboptimality degree $\alpha \in$ $(0,1]$, i.e., for each $\hat{x} \in \mathbb{R}^{n}$ the relaxed Lyapunov inequality

$V_{T}\left(x\left(\delta ; \hat{x}, \bar{u}_{T}^{\star}\right)\right) \leq V_{T}(\hat{x})-\alpha \int_{0}^{\delta} \ell\left(x\left(s ; \hat{x}, \bar{u}_{T}^{\star}\right), \bar{u}_{T}^{\star}(s, \hat{x})\right) d s$

holds with $\bar{u}_{T}^{\star}=\bar{u}_{T}^{\star}(\cdot ; \hat{x})$ defined by Step 2 of Algorithm 1. Then, the suboptimality estimate

$$
\begin{aligned}
J_{\infty}\left(x^{0}, \mu_{T, \delta}^{M P C}\right) & :=\int_{0}^{\infty} \ell\left(x_{\mu_{T, \delta}}^{M P C}\left(t ; x^{0}\right), u_{T, \delta}^{M P C}\left(t ; x^{0}\right)\right) d t \\
& \leq V_{\infty}\left(x^{0}\right) / \alpha
\end{aligned}
$$

holds. If, in addition, conditions (2) and $V_{T}(x) \leq \bar{\eta}(\| x-$ $\left.x^{\star} \|\right)$ hold for some $\mathcal{K}_{\infty}$-function $\bar{\eta}$, the MPC closed loop is asymptotically stable.

The performance (suboptimality) estimate shows that the cost of the MPC closed loop $J_{\infty}\left(x^{0}, \mu_{T, \delta}^{M P C}\right)$ on the infinite horizon is bounded by the infinite horizon optimal cost multiplied by $\alpha^{-1}$. With respect to concluding (asymptotic) stability, the main difficulty is to show the existence of $\alpha>0$ such that the relaxed Lyapunov inequality (3) holds. This point will be investigated more closely in the following subsection.

\section{Controllability Condition}

In the following theorem, a controllability condition is introduced, which allows for verifying validity of (3) based on open loop control functions. Alternatively, bounds on the value function analogously to [16] can be used.

Theorem 2 ([13]) If an overshoot bound $C \geq 1$ and a decay rate $\mu>0$ exist such that, for each $x^{0} \in \mathbb{R}^{n}$, an open loop control function $u_{x^{0}} \in \mathcal{L}_{\text {loc }}^{1}\left(\mathbb{R}_{\geq 0}, \mathbb{U}\right)$ exists satisfying

$$
\ell\left(x\left(t ; x^{0}, u_{x^{0}}(\cdot)\right), u_{x^{0}}(t)\right) \leq C e^{-\mu t} \min _{u \in \mathbb{U}} \ell\left(x^{0}, u\right)
$$

for all $t \geq 0$, then the relaxed Lyapunov inequality (3) holds with performance estimate $\alpha_{T, \delta}$ given by

$$
1-\frac{\sqrt[C]{e^{\mu \delta}-1}}{\sqrt[C]{e^{\mu T}-1}-\sqrt[C]{e^{\mu \delta}-1}} \cdot \frac{\sqrt[C]{e^{\mu(T-\delta)}-1}}{\sqrt[C]{e^{\mu T}-1}-\sqrt[C]{e^{\mu(T-\delta)}-1}} .
$$

Moreover, for given control horizon $\delta$ and a desired performance bound $\bar{\alpha} \in(0,1)$, there always exists $T \in(\delta, \infty)$ such that $\alpha_{T, \delta} \geq \bar{\alpha}$ holds.

Formula (5) allows to easily calculate a prediction horizon $T$ such that $\alpha_{T, \delta}>0$ and, as a direct consequence of Theorem 1, asymptotic stability of the MPC closed loop holds. Furthermore, a certain performance bound $\bar{\alpha}$ can be ensured by choosing a long enough prediction horizon. 


\section{Nonholonomic Integrator Example}

We briefly illustrate the presented results by a nonholonomic integrator example with vector field

$$
f: \mathbb{R}^{3} \times \mathbb{R}^{2} \rightarrow \mathbb{R}^{3}, \quad(x, u) \mapsto\left(u_{1}, u_{2}, x_{1} u_{2}-x_{2} u_{1}\right)^{T},
$$

and stage costs $\ell(x, u)=x_{1}^{2}+x_{2}^{2}+3\left|x_{3}\right|+u_{1}^{2}+u_{2}^{2}$. This system does not admit a continuously differentiable control Lyapunov function and cannot be asymptotically stabilized by continuous state feedback; see [1]. However, for an arbitrary initial state $x^{0} \in \mathbb{R}^{3}$, using the piecewise constant control function $u: \mathbb{R}_{\geq 0} \rightarrow \mathbb{R}^{2}$ defined componentwise by

$$
u_{1}(t)= \begin{cases}-x_{1}^{0} / t_{1}, & t \in\left[0, t_{1}\right) \\ \frac{x_{3}^{0}}{\left|x_{3}^{0}\right|} \cdot \frac{4 \sqrt{\left|x_{3}^{0}\right|}}{\sqrt{2}\left(t_{2}-t_{1}\right)} \sin \left(\left\lfloor\frac{4\left(t-t_{1}\right)}{t_{2}-t_{1}}\right\rfloor \frac{\pi}{2}\right), & t \in\left[t_{1}, t_{2}\right) \\ 0, & t \in\left[t_{2}, \infty\right)\end{cases}
$$

and

$$
u_{2}(t)= \begin{cases}-x_{2}^{0} / t_{1}, & t \in\left[0, t_{1}\right) \\ \frac{4 \sqrt{\left|x_{3}^{0}\right|}}{\sqrt{2}\left(t_{2}-t_{1}\right)} \cos \left(\left\lfloor\frac{4\left(t-t_{1}\right)}{t_{2}-t_{1}}\right\rfloor \frac{\pi}{2}\right), & t \in\left[t_{1}, t_{2}\right) \\ 0, & t \in\left[t_{2}, \infty\right)\end{cases}
$$

steers the system to the desired equilibrium $x^{\star}=0$. While doing so the state trajectory first reaches the $x_{3}$-axis at time $t_{1}$, i.e. $x\left(t_{1}\right)=\left(0,0, x_{3}^{0}\right)^{T}$, and is then driven towards the origin until time $t_{2}$. Calculating the state trajectory for $t_{1}=1$ and $t_{2}=5$ first and plugging the resulting expressions into the stage costs reveals that the controllability condition (4) holds with overshoot bound $C=3$ and decay rate $\mu=0.1$, see [19, Section 2.2] for similar calculation details. Then, for a fixed control horizon $\delta=2$, the performance bound $\alpha_{T, \delta}$ can be evaluated according to Formula (5). Hence, asymptotic stability of the MPC closed loop is ensured for prediction horizon $T \gtrsim 27.5$ as can be seen in Figure 1 (solid line without markers). The employed control $u(\cdot) \in \mathcal{P C}(\mathbb{R}$ geq $0, \mathbb{U}), \mathbb{U}=\mathbb{R}^{m}$, exhibits five discontinuities at $t_{1}+i\left(t_{2}-t_{1}\right) / 4, i \in\{0,1,2,3,4\}$.

\section{SAMPLING AND PERFORMANCE}

While the proposed approach yields satisfactory estimates on the prediction horizon length guaranteeing stability of the MPC closed loop, the proof of Theorem 2 requires that the concatenation of two control functions at an arbitrary time instant $t \in[0, T)$ is again feasible for the minimization in Step 2 of Algorithm 1 even if - as in the case of the nonholonomic integrator - condition (4) can be established for functions, which are piecewise constant on equidistant intervals. In conclusion, the optimization has to be carried out over piecewise continuous (constant) functions. However, the class $\mathcal{P C}([0, T), \mathbb{U})$ is neither well suited for numerically solving the minimization problem nor for implementing the resulting control signal at the plant. For the latter, sampleddata systems with zero order hold are a desirable class of input signals. For sampling period $\tau$, the respective control functions $u$ in $\mathcal{Z} \mathcal{O H}_{\tau}\left([0, T), \mathbb{R}^{m}\right), T=N \tau$ with $N \in \mathbb{N}$, belong to the function space $\mathcal{P C}\left([0, T), \mathbb{R}^{m}\right)$, with the additional property $\left.u(\cdot)\right|_{[i-1) \tau, i \tau)} \equiv \bar{u}_{i} \in \mathbb{R}^{m}, i \in\{1,2, \ldots, N\}$,

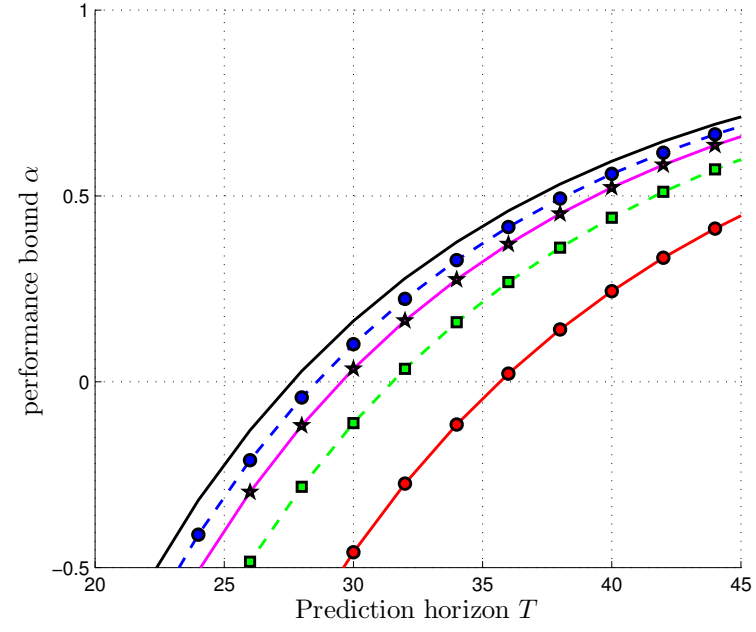

Fig. 1. Performance bounds $\alpha_{T, \delta}, \delta=2$, resulting from Theorems 1 and 2 for the continuous time setting (solid black line). Moreover, the corresponding counterparts for the discrete time setting to be introduced in the following section are also drawn. These depend on a discretization parameter $\tau=2^{-i} \delta, i=0(\circ), i=1(\square), i=2(\star)$, and $i=3(\circ)$.

i.e. piecewise constant except at the sampling instances $i \tau$, $i \in\{1,2, \ldots, N-1\}$. Our goal is to derive stability guarantees for sampled-data systems with zero order hold assuming that the proposed controllability condition can be verified for this function class. To this end, the exact discrete time representation $f_{\tau}: \mathbb{R}^{n} \times \mathbb{R}^{m} \rightarrow \mathbb{R}^{n}$ given by

$$
x(n+1)=f_{\tau}(x(n), u(n))=\Phi(\tau ; x(n), \tilde{u}(\cdot))
$$

of the sampled-data system is considered, where $\tau$ denotes the length of the sampling interval and $\Phi(\tau ; x(n), \tilde{u}(\cdot))$ represents the solution of the differential equation (1) with $\tilde{u}(t) \equiv u(n), t=[0, \tau)$, emanating from initial condition $x(n)$ at time $\tau$; see [9] for details. Here, the discrete time $n$ corresponds to the continuous time $n \tau$ and the control function $\tilde{u}:[0, \tau) \rightarrow \mathbb{R}^{m}$ is identified with the value $u(n) \in \mathbb{R}^{m}$. The "state trajectory" is now a sequence in $\mathbb{R}^{n}$ — denoted by $\left(x_{u}\left(n ; x^{0}\right)\right)_{n=0}^{\infty}$ — for a given sequence of control values $u=(u(n))_{n=0}^{\infty}$ and initial value $x^{0} \in \mathbb{R}^{n}$. The (discrete time) running costs are defined as

$$
\ell_{\tau}(x, u)=\tau \ell(x, u) .
$$

Note that, due to continuity of the state trajectory, this is a good approximation of the continuous time integral cost for $u(\cdot) \in \mathcal{Z} \mathcal{O H}_{\tau}\left([0, T), \mathbb{R}^{m}\right)$, at least for small sampling periods $\tau$.

Remark 3 If the continuous time running costs are separable, i.e., $\ell(x, u)=\ell\left(x, u^{\star}\right)+\ell\left(x^{\star}, u\right)$ holds, the discrete time running costs defined by (6) satisfy $\ell\left(x^{\star}, u\right) \geq 0$ and, thus,

$$
\min _{u \in \mathbb{R}^{m}} \ell_{\tau}(x, u)=\ell_{\tau}\left(x, u^{\star}\right) .
$$

Hence, $\tau \cdot \min _{u \in \mathbb{U}} \ell(x, u)=\min _{u \in \mathbb{R}^{m}} \ell_{\tau}(x, u)$ holds. This separability property holds, e.g. for the running costs used in nonholonomic integrator example or for quadratic 
cost $\ell(x, u)=\left\|x-x^{\star}\right\|^{2}+\lambda\left\|u-u^{\star}\right\|^{2}$. In contrast to that, if the discrete time running costs exactly represent the continuous time cost, i.e. $\ell_{\tau}(x, u)=\int_{0}^{\tau} \ell(\Phi(t ; x, \tilde{u}(\cdot)), \tilde{u}(t)) d t$, another input signal may yield a lower value since the interplay of the control and the current state is taken into account, see [18] for a numerical case study.

Next, we proceed analogously to the continuous time case in order to derive a suboptimality estimate for the considered sampled-data system. To this end, the following discrete time counterpart of Condition (4) is needed.

Assumption 1 Let an overshoot bound $C \geq 1$ and a decay rate $\sigma \in(0,1)$ exist such that, for each state $\hat{x} \in \mathbb{R}^{n}$, there exists a sequence $\left(u_{\hat{x}}(n)\right)_{n=0}^{\infty} \subset \mathbb{R}^{m}$ satisfying the inequality

$$
\ell_{\tau}\left(x_{u_{\hat{x}}}(n ; \hat{x}), u_{\hat{x}}(n)\right) \leq C \sigma^{n} \min _{u \in \mathbb{R}^{m}} \ell_{\tau}(\hat{x}, u) \quad \forall n \in \mathbb{N}_{0} .
$$

Based on Assumption 1, results similar to Theorems 1 and 2 can be derived for discrete time systems, see [4]. These allow to ensure asymptotic stability or a desired suboptimality degree for sampled-data systems with zero order hold if Assumption 1 holds, i.e., the regularity of the function class used in order to verify Inequality (7) is preserved. In particular, the optimal control problem to be solved in Step 2 of Algorithm 1 is now a finite dimensional (nonlinear) optimization problem.

Considering the nonholonomic integrator example once more and taking the structure of the employed control into account shows that Inequality (4) implies Assumption 1 with the same overshoot constant $C$ and decay rate $\sigma:=e^{-\mu \tau}=$ $e^{-\mu \delta}$. However, this leads to more conservative performance bounds, see [13] and Figure 1 (red solid line marked with $\circ)$. Hence, the question arises whether structural properties of control functions used in order to verify Inequality (4) can be preserved while maintaining the superior performance bounds of the continuous time approach.

\section{A. Decoupling of Control Horizon \& Discretization}

A key idea in order to solve this problem is to decouple the control horizon $\delta$ and the discretization parameter $\tau$. To be more precise, the interval $[0, \delta)$ is subdivided into $k \in \mathbb{N}$ (equidistant) sampling intervals $(k \tau=\delta)$. Then, the MPC algorithm is adapted such that the first $k$ control values are implemented on each interval $[n \delta,(n+1) \delta), n \in \mathbb{N}_{0}$, in the discrete setting, so called multistep MPC. Hence, the relation between control horizon $\delta=k \tau$ and prediction horizon $T$ is independent of the discretization accuracy $\tau$. This allows us to apply the results obtained in [4] and, thus, to ensure $u_{T, \delta}^{M P C} \in \mathcal{Z} \mathcal{O H}_{\tau}\left(\mathbb{R}_{\geq 0}, \mathbb{R}^{m}\right)$ for the MPC control $u_{T, \delta}^{M P C}$ since no switches except at the sampling instances are required in the respective proofs.

Definition 1 Let $N \in \mathbb{N}_{\geq 2}$ and $m \in\{1,2, \ldots, N-1\}$ be given. A multistep feedback law is a map $\mu_{N, m}: \mathbb{R}^{n} \times$ $\{0,1, \ldots, m-1\} \rightarrow \mathbb{R}^{m}$ which is applied according to

$$
x_{\mu_{N, m}}(n+1 ; \hat{x})=f_{\tau}\left(x_{\mu_{N, m}}(n ; \hat{x}), \mu_{N, m}(\hat{x}, n)\right),
$$

for $n \in\{0,1, \ldots, m-1\}$ with $x_{\mu_{N, m}}(0 ; \hat{x})=\hat{x}$.
Algorithm 2 yields an MPC multistep feedback law.

Algorithm 2 MPC Algorithm (discrete time)

Given: Prediction and control horizon $N \in \mathbb{N}_{\geq 2}$, $m \in\{1,2, \ldots, N-1\}$, and discretization paramter $\tau \in \mathbb{R}_{>0}$. Set time $n=0$.

1) Measure the current state $\hat{x}=x(n)$ at time $n$.

2) Minimize the finite horizon cost function

$$
J_{N}(\hat{x}, \bar{u}(\cdot))=\sum_{k=0}^{N-1} \ell_{\tau}\left(\bar{x}_{\bar{u}}(k ; \hat{x}), \bar{u}(k)\right)
$$

subject to dynamics $\bar{x}_{\bar{u}}(k+1 ; \hat{x})=f_{\tau}\left(\bar{x}_{\bar{u}}(k ; \hat{x}), \bar{u}(k)\right)$, $\bar{x}_{\bar{u}}(0 ; \hat{x})=\hat{x}$, and $u(k) \in \mathbb{U}, k \in\{0,1, \ldots, N-1\}$ to compute an optimal sequence $\left(\bar{u}_{N}^{\star}(k ; \hat{x})\right)_{k=0}^{N-1} \subset \mathbb{R}^{m}$.

3) Implement $\left(\bar{u}_{N}^{\star}(k ; \hat{x})\right)_{k=0}^{m-1}$ at the plant, set $n=n+m$ and goto Step 1 .

The MPC multistep feedback is defined by $\mu_{N, m}(\hat{x}, k)=$ $\bar{u}_{N}^{\star}(k ; \hat{x})$ for $k=0,1, \ldots, m-1$. "Classical" MPC as used in literature corresponds to $m=1$.

\section{B. Discretizations}

We want to rigorously investigate the interplay between faster sampling and the performance bound $\alpha$. To this end, the following definition is needed in order to investigate whether a finer discretization may close the observed gap if the control horizon $\delta=\tau m$ is kept constant.

\section{Definition 2 (Discretization and Iterative Refinement)}

Let $\tau_{0} \in(0, \delta]$ be chosen such that the conditions $m:=\delta / \tau_{0} \in \mathbb{N}$ and $N:=T / \tau_{0} \in \mathbb{N}$ hold. In addition, let a decay rate $\sigma$ be given and assume that the overshoot $C$ does not depend on the discretization parameter $\tau$. Furthermore, let a sequence $\left(k_{j}\right)_{j \in \mathbb{N}_{0}} \subseteq \mathbb{N}$ with $k_{0}:=1$ be given. Then, a discretization $\mathcal{D}$ is defined by a sequence of quintruplets

$$
\begin{aligned}
\mathcal{D}=\left(\mathcal{D}_{j}\right)_{j \in \mathbb{N}_{0}} & =\left(k_{j}, \tau_{j}, N_{j}, m_{j}, \sigma_{j}\right)_{j \in \mathbb{N}_{0}} \\
& :=\left(k_{j}, k_{j}^{-1} \tau_{0}, k_{j} N, k_{j} m, \sigma^{1 / k_{j}}\right)_{j \in \mathbb{N}_{0}} .
\end{aligned}
$$

Here, $\left(\tau_{j}\right)_{j \in \mathbb{N}_{0}}, \tau_{j}:=\tau_{0} / k_{j}$, represents the sequence of discretization parameters (i.e., the sampling time steps) and the parameters $\left(N_{j}\right)_{j \in \mathbb{N}_{0}}$ and $\left(m_{j}\right)_{j \in \mathbb{N}_{0}}$ specify the feedback laws $\left(\mu_{N_{j}, m_{j}}\right)_{j \in \mathbb{N}_{0}}$. If $k_{j+1}$ is a multiple of $k_{j}$ and $k_{j+1}>k_{j}$ holds, a sequence $\left(k_{j}\right)_{j \in \mathbb{N}_{0}} \subset \mathbb{N}$ is called an iterative refinement.

In the $j$-th discretization the interval $\left[0, \tau_{0}\right)$ is subdivided into $k_{j}$ equidistant parts, i.e.,

$$
\left[0, \tau_{0} / k_{j}\right),\left[\tau_{0} / k_{j}, 2 \tau_{0} / k_{j}\right), \ldots,\left[\left(k_{j}-1\right) \tau_{0} / k_{j}, \tau_{0}\right) .
$$

\section{Application to the Nonholonomic Integrator Example}

As shown before the controllability Assumption 1 is preserved with the same overshoot bound $C$ and decay rate $\sigma=e^{-\mu \tau}$ for the nonholonomic integrator example. However, now the sampling rate $\tau$ is decoupled from the control horizon $\delta$. More precisely, $m$ control values are implemented at the plant (Step 3 of Algorithm 2) before a 
reoptimization is carried out (Step 2 of Algorithm 2). Here, the following stability theorem, which was proven in [4], is applied in order to determine the suboptimality degree $\alpha_{N, m}$.

Theorem 3 Let $N \in \mathbb{N}_{\geq 2}, m \in\{1,2, \ldots, N-1\}$, and $\bar{\alpha} \in(0,1)$ be given as well as Assumption 1 be satisfied with $C \geq 1$ and $\sigma \in(0,1)$. Furthermore, assume that $\mathcal{K}_{\infty}$ functions $\widetilde{\alpha}_{1}(\cdot)$, $\widetilde{\alpha}_{2}(\cdot)$ exist satisfying

$$
\widetilde{\alpha}_{1}\left(\left\|x-x^{\star}\right\|\right) \leq \ell_{\tau}\left(x, u^{\star}\right) \leq \widetilde{\alpha}_{2}\left(\left\|x-x^{\star}\right\|\right)
$$

for all $x \in \mathbb{R}^{n}$. In addition, let the inequality $\alpha_{N, m} \geq \bar{\alpha}$ hold with $\alpha_{N, m}$ given by

$$
1-\frac{\prod_{i=m+1}^{N}\left(\gamma_{i}-1\right) \prod_{i=N-m+1}^{N}\left(\gamma_{i}-1\right)}{\left[\prod_{i=m+1}^{N} \gamma_{i}-\prod_{i=m+1}^{N}\left(\gamma_{i}-1\right)\right]\left[\prod_{i=N-m+1}^{N} \gamma_{i}-\prod_{i=N-m+1}^{N}\left(\gamma_{i}-1\right)\right]} .
$$

with $\gamma_{i}:=C \sum_{n=0}^{i-1} \sigma^{i}=C\left(1-\sigma^{i}\right) /(1-\sigma)$. Then, for each $x^{0} \in \mathbb{R}^{n}$, the MPC closed loop $x_{\mu_{N, m}}^{M P C}\left(\cdot ; x^{0}\right)$ is asymptotically stable and its costs

$J_{\infty}\left(x_{0}, \mu_{N, m}^{M P C}\left(\cdot ; x_{0}\right)\right)=\sum_{n=0}^{\infty} \ell_{\tau}\left(x_{\mu_{N, m}}^{M P C}\left(n ; x_{0}\right), u_{N, m}^{M P C}\left(n ; x_{0}\right)\right)$ satisfy the estimate $J_{\infty}\left(x_{0}, u_{N, m}^{M P C}\left(\cdot ; x_{0}\right)\right) \leq \bar{\alpha}^{-1} \cdot V_{\infty}\left(x_{0}\right)$.

Using $\alpha_{N, m}$ defined by Formula (9) and coupling the control horizon $\delta$ and the discretization parameter $\tau$ as in Definition 2 yields suboptimality degrees in dependence of the discretization accuracy $\tau$. For $\tau=2^{-i} \delta, i \in\{0,1,2,3\}$, the resulting performance bounds are illustrated in Figure 1. The finer the discretization, the better the suboptimality bounds. Indeed, monotone convergence to the continuous time values $\alpha_{T, \delta}$ can be observed in this example. In the following section, we rigorously formulate this observation as a theorem.

\section{Main Result}

We show that structural properties of the control functions used to verify Assumption 1 can be preserved while the superior continuous time estimates on performance and, thus, the length of a stabilizing prediction horizon are maintained. First, all assertions dealing with the discretized setting are presented in Proposition 1. Then, in Theorem 4, conclusions on the connection to the continuous time performance bounds are drawn. To this end, let the expression

$$
\gamma_{i, k}:=C \sum_{n=0}^{i-1} \sigma_{k}^{n}=C \sum_{n=0}^{i-1}\left(\sigma^{1 / k}\right)^{n}=\frac{C\left(1-\sigma^{i / k}\right)}{1-\sigma^{1 / k}}
$$

be defined in dependence of the parameter $k \in \mathbb{N}_{0}$ and denote $\alpha_{N, m}$ from Formula (9) based on $C$ and $\sigma_{k}=\sigma^{1 / k}, k \in$ $\mathbb{N}$, by $\alpha_{k N, k m}(k)$, i.e., the argument $k$ indicates that $\gamma_{i}$ is replaced by $\gamma_{i, k}$.

Proposition 1 (Monotone Convergence) Let a discretization $\mathcal{D}=\left(\mathcal{D}_{j}\right)_{j \in \mathbb{N}_{0}}=\left(k_{j}, \tau_{j}, N_{j}, m_{j}, \sigma_{j}\right)_{j \in \mathbb{N}_{0}}$ according to Definition 2 with $N \in \mathbb{N}_{\geq 2}, m \in\{1,2, \ldots, N-1\}$, and $k_{j} \rightarrow \infty$ for $j$ tending to infinity be given. Suppose, in addition, that for each discretization parameter $\tau_{j}, j \in \mathbb{N}_{0}$, Assumption 1 is satisfied with decay rate $\sigma_{j}=\sigma^{k_{j}^{-1}}, \sigma \in$ $(0,1)$, and overshoot $C \geq 1$. Then, we get convergence of $\alpha_{k_{j} N, k_{j} m}\left(k_{j}\right)$ to

$1-\frac{\left(\frac{1-\sigma^{m}}{\sigma^{m}}\right)^{1 / C}}{\left(\frac{1-\sigma^{N}}{\sigma^{N}}\right)^{1 / C}-\left(\frac{1-\sigma^{m}}{\sigma^{m}}\right)^{1 / C}} \cdot \frac{\left(\frac{1-\sigma^{N-m}}{\sigma^{N-m}}\right)^{1 / C}}{\left(\frac{1-\sigma^{N}}{\sigma^{N}}\right)^{1 / C}-\left(\frac{1-\sigma^{N-m}}{\sigma^{N-m}}\right)^{1 / C}}$

for $j \rightarrow \infty$. Moreover, for iterative refinements, i.e. $k_{j}$ is a multiple of $k_{j-1}$ for all $j \in \mathbb{N}$, the sequence $\left(\alpha_{k_{j} N, k_{j} m}\left(k_{j}\right)\right)_{j \in \mathbb{N}_{0}}$ is monotonically increasing.

Proposition 1 shows that finer discretizations lead to improved suboptimality bounds, i.e.

$$
\alpha_{k N, k m}(k) \leq \alpha_{\nu k N, \nu k m}(\nu k) \quad \forall k, \nu \in \mathbb{N},
$$

holds. This property can be employed in order to ensure, e.g., asymptotic stability of the MPC closed loop for shorter prediction horizons. Moreover, we computed the limit for discretization parameter $\tau$ tending to zero $(k \rightarrow \infty)$. Combining these results allows for connecting the structure preserving estimates obtained for discretized systems with the superior performance estimates derived in the purely continuous time setting. Indeed, the following theorem is almost a direct consequence of Proposition 1.

Theorem 4 Let the assumptions of Proposition 1 hold. If, in addition, Condition (4) holds with the same overshoot constant $C$ and such that the decay rate satisfies the condition $\sigma=e^{-\mu \tau_{0}}$, then we get

$$
\lim _{j \rightarrow \infty} \alpha_{k_{j} N_{0}, k_{j} m_{0}}\left(k_{j}\right)=\alpha_{T, \delta}
$$

with $\alpha_{T, \delta}$ from Formula (5), $\delta:=m \tau_{0}$, and $T:=N \tau_{0}$, respectively. Moreover, the suboptimality degree $\alpha_{T, \delta}$ of the continuous setting is an upper bound, i.e., $\alpha_{T, \delta} \geq$ $\alpha_{k N_{0}, k m_{0}}(k)$ holds for all $k \in \mathbb{N}$.

In [19, Theorem 3.2, Corollary 3.4], we proved that the discrete time performance estimates converge for discretization parameter $\tau$ tending to zero $(k \rightarrow \infty)$ to their upper bound given by $\alpha_{T, \delta}$ if the control and prediction horizon, i.e. $\delta=\tau_{j} m_{j}$ and $T=\tau_{j} N_{j}$ for all $j \in \mathbb{N}_{0}$, are kept constant. For iteratively refined discretizations, this convergence was shown to be monotone, cf. [19, Proposition 3.3].

Theorem 4 mainly requires that the continuous time (exponential) controllability condition (4) and its discrete time counterpart Assumption 1 are consistent, i.e., hold with the same overshoot bound $C$ and the decay rate fulfils $\sigma=$ $e^{-\mu \tau_{0}}$. In this case, Theorem 4 shows the connection between MPC without stabilizing constraints for the continuous and the discrete time setting. Here, the continuous time results can be interpreted as limit case of what is achievable for sufficiently fast sampling. Hence, using multistep feedback laws, a sampling period (discretization accuracy) can be determined such that the guaranteed performance estimate is arbitrarily well recovered. In particular, this allows to derive stability guarantees for sampled-data systems with zero order hold. 
The assertions of Proposition 1 and Theorem 4 are illustrated in Figure 2 for an iterative refinement. The discrete time estimates approximate their continuous time counterpart already after a few refinements very well. This observation is supported by the illustration drawn on the right which shows that the discretization error decays exponentially.
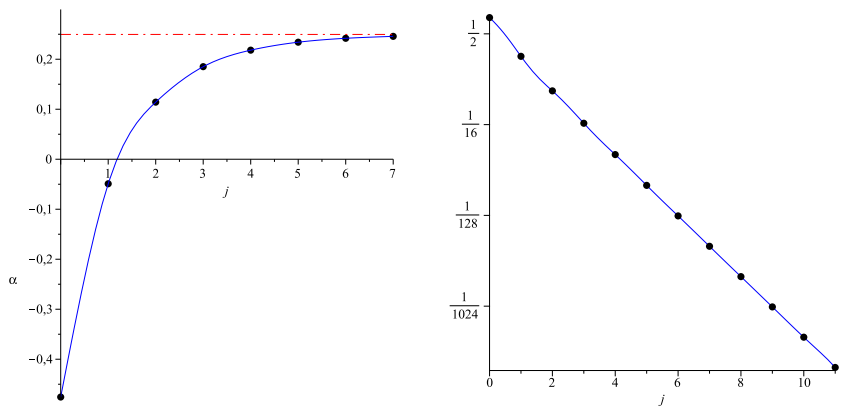

Fig. 2. The figure on the left dipicts the monotone convergence of $\left(\alpha_{k_{j} N, k_{j} m}\left(k_{j}\right)\right)_{j \in \mathbb{N}_{0}}$ for $k_{j}=2^{j}$ to the limit stated in Proposition 1. On the right, the deviations from the theoretically obtained upper bound are depicted. Here, we choose $N=4, m=1, \tau=1, C=2$, and $\sigma=0.5$.

Remark 4 Sampled-data systems with zero order hold use control functions, which are piecewise constant on each sampling interval. Hence, the OCP in Step 2 of the MPC algorithm breaks down to a finite dimensional (nonlinear) optimization problem, which is significantly easier to solve. However, the number of optimization variables rapidly grows for faster sampling (finer discretizations). Hence, the presented results give insight into the trade-off between slow sampling and guaranteed performance.

The discussed procedure for choosing the relevant parameters for MPC can be summarized as follows. First, the controllability condition (4) has to be verified for an overshoot bound $C$ and a decay rate $\mu$. Second, the continuous time performance estimate $\alpha_{T, \delta}$ in (5) allows to determine a prediction horizon length for which asymptotic stability and a certain performance of the MPC closed loop is guaranteed in the continuous time case. Finally, a sampling rate can be determined such that asymptotic stability and satisfactory performance are achieved (possibly arbitrarily close to the upper bound $\alpha_{T, \delta}$ of the continuous time approach). In the case that this sampling rate is not feasible, e.g., due to hardware limitations, the prediction horizon might have to be increased in a follow-up step in order to recover performance of the closed loop despite slower sampling.

\section{CONCLUSIONS}

In this paper, we elaborated on a structure preserving property of performance estimates for discrete time systems. This feature can in particular be used in order to ensure asymptotic stability of the MPC closed loop for sampled-data systems with zero order hold. Our research was motivated by the fact that estimates resulting from the purely continuous time approach [13] yield better bounds for the required length of the prediction (optimization) horizon. We presented a framework in which the advantages of both techniques are merged such that asymptotic stability can be guaranteed for control functions, which are piecewise constant on a priori fixed (equidistant) intervals while the suboptimality degree from the continuous time approach is maintained.

\section{REFERENCES}

[1] R. W. Brockett. Asymptotic stability and feedback stabilization. In R. W. Brockett, R. S. Millman, and H. J. Sussmann, editors, Differential Geometric Control Theory, pages 181-191. Birkhäuser, Boston, 1983

[2] H. Chen and F. Allgöwer. A quasi-infinite horizon nonlinear model predictive control scheme with guaranteed stability. Automatica, 34(10):1205-1218, 1998.

[3] L. Grüne and J. Pannek. Nonlinear Model Predictive Control: Theory and Algorithms. Communications and Control Engineering. Springer London Dordrecht Heidelberg New York, 2011.

[4] L. Grüne, J. Pannek, M. Seehafer, and K. Worthmann. Analysis of unconstrained nonlinear MPC schemes with time-varying control horizon. SIAM J. Control Optim., 48(8):4938-4962, 2010.

[5] L. Grüne and A. Rantzer. On the infinite horizon performance of receding horizon controllers. IEEE Transactions on Automatic Control, 53(9):2100-2111, 2008.

[6] A. Jadbabaie, J. Yu, and J. Hauser. Unconstrained Receding-Horizon Control of Nonlinear Systems. IEEE Transactions on Automatic Control, 46(5):776-783, 2001.

[7] H. Logemann and E.P. Ryan. Ordinary Differential Equations Analysis, Qualitative Theory and Control. Springer Undergraduate Mathematics Series. Springer London Heidelberg New York Dordrecht, 2014.

[8] V. Nevistić and J. Primbs. Receding Horizon Quadratic Optimal Control: Performance Bounds for a Finite Horizon Strategy. In 1997 European Control Conference, Brussels, Belgium, 1997.

[9] D. Nešić and A.R. Teel. A framework for stabilization of nonlinear sampled-data systems based on their approximate discrete-time models. IEEE Transactions on Automatic Control, 49(7):1103-1122, 2004.

[10] S. J. Qin and T. A. Badgwell. A survey of industrial model predictive control technology. Control Engineering Practice, 11(7):733-764, 2003

[11] T. Raff, S. Huber, Z. K. Nagy, and F. Allgöwer. Nonlinear model predictive control of a four tank system: An experimental stability study. In Proceedings of the IEEE Conference on Control Applications, pages 237-242, Munich, Germany, 2006.

[12] J. B. Rawlings and D. Q. Mayne. Model Predictive Control: Theory and Design. Nob Hill Publishing, Madison, WI, USA, 2009.

[13] M. Reble and F. Allgöwer. Unconstrained model predictive control and suboptimality estimates for nonlinear continuous-time systems. Automatica, 48(8):1812-1817, 2012.

[14] E.D. Sontag. Mathematical Control Theory - Deterministic Finite Dimensional Systems. Number 6 in Texts in Applied Mathematics. Springer-Verlag New York, second edition, 1998.

[15] Michael A Stephens, Chris Manzie, and Malcolm C Good. Model predictive control for reference tracking on an industrial machine tool servo drive. Industrial Informatics, IEEE Transactions on, 9(2):808816, 2013.

[16] S. E. Tuna, M. J. Messina, and A. R. Teel. Shorter horizons for model predictive control. In Proceedings of the American Control Conference, Minneapolis, Minnesota, USA, 2006.

[17] Sergio Vazquez, J Leon, L Franquelo, Jose Rodriguez, H Young, Abraham Marquez, and Pericle Zanchetta. Model predictive control: A review of its applications in power electronics. Industrial Electronics Magazine, IEEE, 8(1):16-31, 2014.

[18] K. Worthmann. Estimates of the Prediction Horizon Length in MPC a Numerical Case Study. In Proceedings of the IFAC Conference on Nonlinear Model Predictive Control 2012 (NMPC'12), Noordwijkerhout, the Netherlands, page 0037.pdf, 2012.

[19] K. Worthmann, M. Reble, L. Grüne, and F. Allgöwer. The Role of Sampling for Stability and Performance in Unconstrained Model Predictive Control. SIAM J. Control Optim., 52(1):581-605, 2014 